\title{
Erratum to: Discussions on Hirsch Conjecture and Existence of Strongly Polynomial-time Simplex Variants
}

\section{Pei-Zhuang Wang}

\section{Erratum to: Ann. Data. Sci. (2014) 1(1):41-71 DOI 10.1007/s40745-014-0005-9}

In the original published version of this article, several errors are unfortunately found. The illustrations and corrections of these errors are listed as below:

\section{Title}

Discussions on Hirsch Conjecture and The Existence of Strongly Polynomial: Time Simplex Variants

Correction:

Discussions on Hirsch Conjecture and Existence of Strongly Polynomial-time Simplex Variants

2. The row of Example 4.1 in page 59:

Example 4.1. Given ${ }^{0} \boldsymbol{g}=-\boldsymbol{b}^{T}=(-4,-1,-4,-6,-2), F_{0}=(7,4,7,6,5) \in$ Correction:

Example 4.1. Given ${ }^{0} \boldsymbol{g}=-\boldsymbol{b}^{T}=(-4,-1,-4,-6,-2), F_{0}=(7,4,7,9,5) \in$

3. The rows from second row to fifth row, under the tableau in page 66:

$k=0 . \Delta=\varnothing$, there is no door at $F_{0}=(7,4,7,6,5)$, go to Step 3,

The online version of the original article can be found under doi:10.1007/s40745-014-0005-9.

\footnotetext{
P.-Z. Wang $(\varangle)$

College of Science, Liaoning Engineering Technology Univ., Liaoning 123000, Fuxin, China e-mail: peizhuangw@126.com

P.-Z. Wang

Research Center of Fictitious Economy and Data Science, Chinese Academy of Sciences, Beijing 100080, China
} 
Step 3 Getting next stage point and the door cone $C_{1}$ :

$$
\begin{aligned}
F_{1} & =F_{k+1}=\text { NextStagePoint }\left(F_{k}, \underline{\boldsymbol{g}}_{\downarrow \ldots(k)}\right) \\
& =\text { NextStagePoint }\left(F_{0},{ }^{0} \boldsymbol{g}\right)=(3,3,3,3) ;
\end{aligned}
$$

\section{Correction:}

$k=0 . \Delta=\varnothing$, there is no door at $F_{0}=(7,4,7,9,5)$, go to Step 3,

Step 3 Getting next stage point and the door cone $C_{1}$ :

$$
\begin{aligned}
F_{1} & =F_{k+1}=\text { NextStagePoint }\left(F_{k}, \underline{\boldsymbol{g}}_{\downarrow \ldots(k)}\right) \\
& =\text { NextStagePoint }\left(F_{0},{ }^{0} \boldsymbol{g}\right)=(3,3,3,3,3) ;
\end{aligned}
$$

